\title{
Motion Prediction in Rock Scissor Paper Game based on Machine Learning
}

\author{
Earl Kim, Jang Yeol Lee, Jun Heon Kim, Kee Hyun Choi, Dong Ryeol Shin
}

\begin{abstract}
The main aim of this system is to predict and analyze gesture pattern from a user based on machine learning. The system is adopted in the rock-paper-scissors game which is suggested as always win at the game by predicting user gesture. Quantization and processing of user gesture from EMG sensor are implemented to generate training data in order that disciplining machine. As suggested procedure, an enormous amount of user gesture data will be collected and training model will be implemented with machine learning. By adopting the implemented system into the game, the research will verify that it is feasible to predict user gesture during playing game. The manner of a game is that computer shows the result when the user starts the rock-paper-scissors game in front of the monitor and the system always shows winning result that is the main purpose of it.
\end{abstract}

Keywords- machine learning, apache spark, streaming, gesture, prediction

\section{Introduction}

In the early stage of big data, most researchers were likely conducted by accumulating large quantities of data and analyzing those. Meanwhile, a recent trend has the purpose to predictively analyze and implement patterning data with machine learning beyond simply analyzing bulk of data.

In this study, real-time data analysis will be performed by patterning various gestures from users with machine learning. Existing gesture recognition has been processed as the basis of image processing using a camera. The problems with this approach are that there is restriction on the area; users should be located in viewing angle of the camera, and on the equipment needed several tools.

Earl Kim

Sungkyunkwan University

South Korea

Jang Yeol Lee

Sungkyunkwan University

South Korea

Jun Heon Kim

Sungkyunkwan University

South Korea

Kee Hyun Choi

Sungkyunkwan University

South Korea

Dong Ryeol Shin

Sungkyunkwan University

South Korea
To overcome these thresholds, firstly, gesture data will be adopted after data digitization by using EMG(electromyogram) sensor. The research will conduct computer training with numerical gesture data and machine learning algorithm and propose the system which is the utilization of the trained computer so that predict user gesture ahead. To test and verify the proposed system, the machine; feasible to implement the rock-paper-scissors game with human, would be given a demonstration. The study suggests that the machine could collect and analyze EMG data from a user in real-time while the user who wear EMG sensor, play the rock-paper-scissors game in front of the monitor according to a rule. The system will definitely win the game and show the result on a screen by predicting user gesture beforehand based on training model. Massive EMG sensor data as to rock-paper-scissors game and big data technique including real-time data collection, machine learning algorithm and so forth are required to realize the system.

\section{Related Work}

\section{A. Apache Flume}

Apache Flume is a framework for collecting data and possible to up gather various log data and transfer data in real-time. Flume has been designed having the core of the reliability, scalability. and manageability of the system.[1].

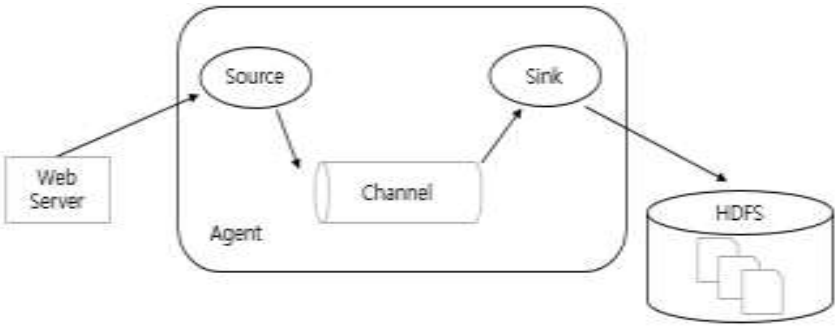

Figure 1. Apache Flume Flow diagram

Flume is composed of the source, channel, and sink as above figure. Source collect log from the web server, Channel saves data temporarily before delivering data to Sink and Sink takes charge of saving data in local or storage such as HDFS and HBase.

There are several source types containing Avro type connecting over agents, NetCat type for TCP communication, Syslog and Exec type. Channel saves received data be the way of one of type among Memory, JDBC, and File. Sink transfers received data to HDFS, HBase, File_Rolll or another agent thereby being able to save received data in intended storage. 


\section{B. HDFS}

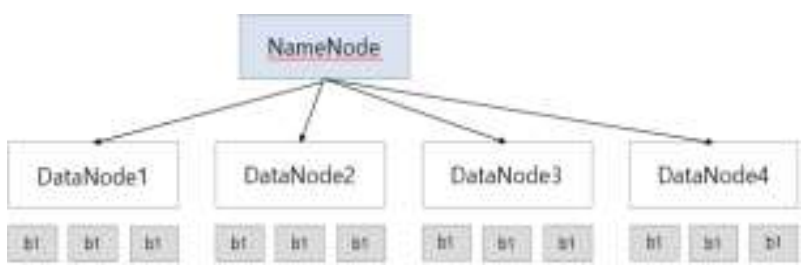

Figure 2. HDFS Architecture

HDFS is a file system to save data in order that distributed data processing provided from Apache institution. Structure of HDFS is composed as the figure2.

In the figure, Namenode, a sort of Master node saving metadata as to file, store up directory structure, varieties of metadata and location where physical file is laid up. An attribute that name node has information regarding all of the data block shall be the cause of SFP(single failure point) that error occurs in all HDFS system if there are the event of the error in name node. Datanode reads virtual files and conducts saving one file with dividing each unit called block. It allows block information which is saved by periodically communicating with name node, to save in name node.

In the figure, a block indicates a minimum unit that carries out reading and writing in HDFS. One file is saved being divided into the various block. The block size of Hadoop adopts bigger block size than that of the general file system; a few kilobytes - generally being $512 \mathrm{~KB}$ in the file system. Hadoop commonly uses $128 \mathrm{MB}$ and $64 \mathrm{MB}$ as a default.

\section{Apache Spark}

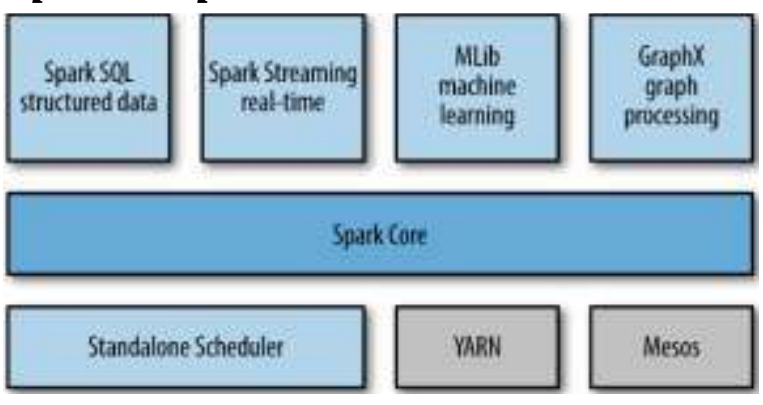

Figure 3. Apache Spark Architecture

Apache Spark is ANOVA system operating on memory. Spark supports various languages such as Java, Scala, Python and R. The strength of Spark is that velocity of MapReduce from Spark is far better than that from Hadoop. Saving input-output and middle data in In-memory as a form of RDD(Resilient Distributed Dataset) facilitates more rapid processing speed because it could show high performance and rapid processing of conversational work road without additional cost or repetition of I/O. [2]

Above figure shows a structure of Stack. There are standalone, Scheduler, YARN and Mesos for operating Spark in infraclass. Spark Core is placed above it because Spark distribution cluster is based on memory.

\section{Machine Learning Algorithm}

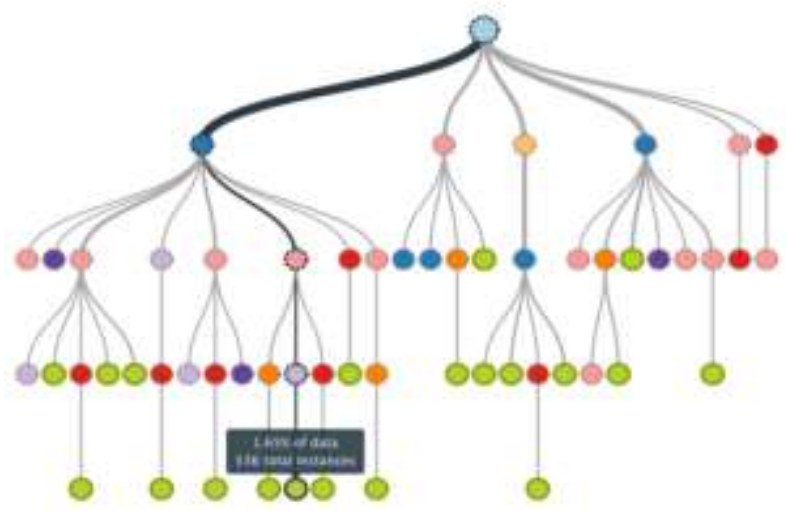

Figure 4. Decision Tree Algorithm

Decision tree model is a powerful and non-probability algorithm which capture more complicated non-linear pattern and feature communication than other general classification model.[3] Decision tree is conducted with various tasks, relatively easy to be translated and understood and can process characteristics of category and digit.

It rarely requires more data to expend its scale and is commensurate with containing ensemble method. All of part from decision tree has class allocated 0 or 1 and all aggregation of features consist one tree. The algorithm of decision tree is top-down way started from root node and chose the characteristic which provides data collection

\section{System design proposal}

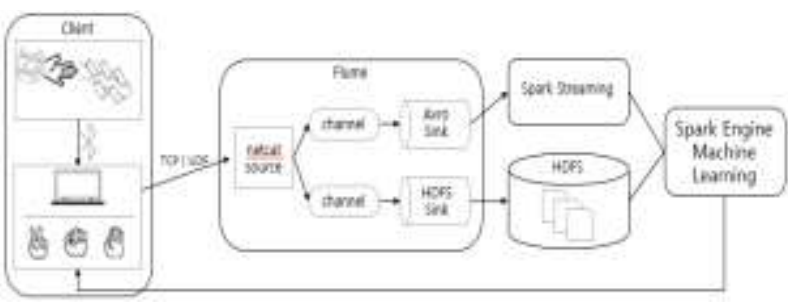

Figure 5. System Design Suggestion

Above figure5 is an overall system block diagram suggested by the study. The overall system conducts data processing for machine learning after collecting rock-paperscissors gesture data from the user who wears equipment with several EMG sensor.[4] The game system should cognize certain gesture with only part of the gesture because it has a function of predicting gesture from users and show its card at the same time; users do. For realizing this, the research should select specific data length within the whole of data length which would be used for discerning gesture data and implement converting data formation for machine learning.[5] Furthermore, a large amount of data as to rockpaper-scissors gesture are required to the more accurate recognition of user gesture. Data will be processed as the formation in below figure6. 


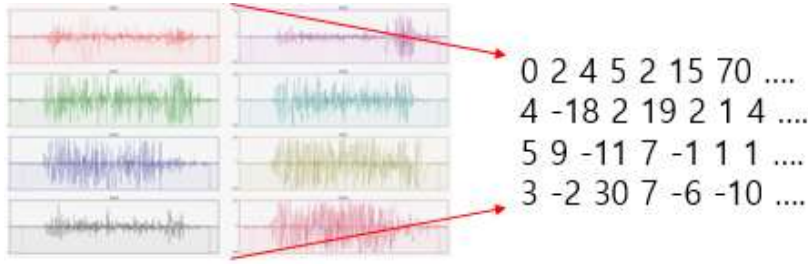

Figure 6. Data Preprocessing

Decision tree model is created by converting processed data to training data set as the figure6. Generated model would be used to analyze and predict gesture data brought from the user. With the proposed training model, the game system suggested in this study conducts quantification and processing of data regarding rock-paper-scissors gesture by adopting EMG sensor. Processed data are transferred to apache flume in real-time via TCP communication. Flume builds up two channels so that one channel transmits data to spark streaming and the other one saves data in HDFS.[6] In spark streaming, it predicts the result of user gesture by using training model created beforehand, and shows the result in a monitor that it will always beat the user. Data accumulated at HDFS in real-time shall create new training model when it comes to constant size, could pursue continuous improvement of rate of data recognition.

\section{Conclusion}

The study suggests the solution and system predict and analyze gesture data. Training circumstance is constructed via machine learning in the system, contrary to data recognition in the existing system. The main purpose of a study is predicting what user shows in real-time, and rockpaper-scissors game system will be tested to verify it. This experiment and research, verified that machine can predict future data by learning user gesture, will be adopted in various motion recognition field.

\section{Acknowledgment}

This research was supported by Basic Science Research Program through the National Research Foundation of Korea(NRF) funded by the Ministry of Education(NRF2016R1A6A3A11930831)

\section{References}

[1] Liu, Xiufeng, Nadeem Iftikhar, and Xike Xie. "Survey of real-time processing systems for big data." Proceedings of the 18th International Database Engineering \& Applications Symposium. ACM, 2014.

[2] Han, Zhijie, and Yujie Zhang. "Spark: A Big Data Processing Platform Based on Memory Computing." Parallel Architectures, Algorithms and Programming (PAAP), 2015 Seventh International Symposium on. IEEE, 2015.

[3] Fu, Jian, Junwei Sun, and Kaiyuan Wang. "SPARK-A Big Data Processing Platform for Machine Learning." Industrial InformaticsComputing Technology, Intelligent Technology, Industrial Information Integration (ICIICII), 2016 International Conference on. IEEE, 2016.

[4] Benatti, S., et al. "EMG-based hand gesture recognition with flexible analog front end." Biomedical Circuits and Systems Conference (BioCAS), 2014 IEEE. IEEE, 2014.

[5] Bhattacharya, Sambit, Bogdan Czejdo, and Nicolas Perez. "Gesture classification with machine learning using kinect sensor data." Emerging Applications of Information Technology (EAIT), 2012 Third International Conference on. IEEE, 2012.
[6] Maarala, Altti Ilari, et al. "Low latency analytics for streaming traffic data with Apache Spark." Big Data (Big Data), 2015 IEEE International Conference on. IEEE, 2015.

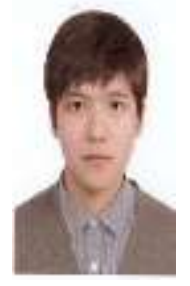

Earl Kim received the B.S degree in the Electronic Engineering from Kyung-Hee University, Korea, in 2015. Currently, he is working for M.S. in Department of Computer Science and Engineering at Sungkyunkwan University, Korea. His research interests include Machine learning and Big data.

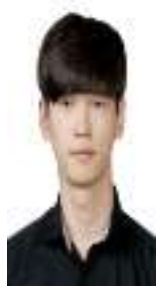

Jang Yeol Lee received the B.S degree in the Information Communication from Korea Nazarene University, Korea, in 2017. Currently, he is working for M.S. in Department of Computer Science and Engineering at Sungkyunkwan University, Korea. His research interests include Machine learning and Big data.

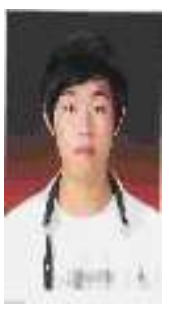

Jun Heon Kim received the B.S degree in the Information \& Communication Engineering from Sung-Kyul University, Korea, in 2016. Currently, he is working for M.S. in Department of Computer Science and Engineering at Sungkyunkwan University, Korea. His research interests include Machine learning and Big data.

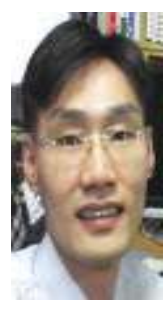

Kee Hyun Choi received the Ph.D. degree in the Electrical, Electronic \& Computer Engineering from Sungkyunkwan University, Korea, in 2005. Currently, he is working for the Dept. of Software, College of Software, Sungkyunkwan University, Korea as a Research Professor (Research Fellow). Interest areas include WiFi MAC, P2P, AQM and IoT.

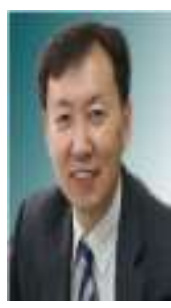

Dong Ryeol Shin received the B.S., M.S., and Ph.D. degrees in Electrical Engineering from the Sungkyunkwan University in 1980, the Korea Advanced Institute of Science and Technology (KAIST) in 1982, and the Georgia Institute of Technology in 1992, respectively. During 1992-1994, he had worked for Samsung Data Systems, Korea, where he was involved in the research of Intelligent Transportation Systems. Since 1994, he has been with the Department of Computer Science and Engineering at Sungkyunkwan University where he is currently a Professor in Network Research Group. His current research interests lie in the areas of mobile network, ubiquitous computing, cloud computing, and bioinformatics. And he is actively involved in the security of vehicular area networks, and the implementation and analysis of big data platform, applicable to 3D image processing of robotic armo 